\title{
Espacios de contención y resiliencia en comunidades educativas frente al contexto de la COVID-19 en Chile
}

\author{
Verónica Hurtubia Toro ${ }^{1}$ (D) \\ Viviana Tartakowsky ${ }^{2}$ (i) \\ Marcelo Acuña ${ }^{2}$ (D) \\ Marta Landoni ${ }^{1}$ (D) \\ ${ }^{1}$ Universita Cattolica del Sacro Cuore, Milán. Italia; ' Universidad Bernardo O'Higgins, Santiago, Chile.
}

Resumen. El objetivo de este estudio, de corte cualitativo, es identificar factores de resiliencia en la comunidad escolar en el contexto de la pandemia COVID-19, frente al cual las comunidades escolares han adaptado sus formas de comunicación y aprendizaje, intensificando responsabilidades y la carga laboral. Los docentes han desarrollado cuadros depresivos, sentimientos de frustración, pérdida del sentido de su labor y una baja percepción de autoeficacia. Como resultado de este escenario, se crearon instancias de apoyo tecnológico y emocional para las comunidades educativas, como el programa "Me cuido para cuidarnos", que involucró a 35 establecimientos educativos particulares y particulares-subvencionados en Chile, alcanzando 1.599 docentes con un total de 259 horas de formación online. El análisis de los datos recolectados por medio de actividades creativo-expresivas arrojan 22 códigos, agrupados en 5 categorías: comunidad, soporte, cuidado y co-cuidado, mejora de espacios y seguridad. Los resultados apuntan a la generación de procesos de resiliencia, al adaptar y generar dentro del mismo contexto escolar los recursos para hacer frente a la incertidumbre. Se torna relevante la continuidad de espacios de apoyo emocional para los docentes, considerando que aún se desconocen las consecuencias psicológicas que tendrá este despliegue de energías en la comunidad escolar.

Palabras clave: resiliencia; covid-19; confinamiento; educación; salud mental.

Espaços de contenção e resiliência em comunidades educativas por influência do contexto do Covid-19 no Chile

Resumo. O objetivo deste estudo, de cunho qualitativo, é identificar fatores de resiliência na comunidade escolar no contexto da pandemia COVID-19, em vista da qual as comunidades escolares adaptaram as suas formas de comunicação e aprendizagem, intensificando responsabilidades e carga de trabalho. Os docentes manifestaram quadros depressivos, sentimentos de frustração, não veem sentido no seu trabalho e possuem baixa percepção de autoeficácia. Como resultado deste cenário foram criadas instâncias de suporte tecnológico e emocional para as comunidades educativas, como o programa "Me cuido para nos cuidarmos" que envolveu 35 estabelecimentos educativos particulares e particulares subsidiados no Chile, alcançando 1.599 docentes somando um total de 259 horas de formação online. A análise dos dados coletados por meio de atividades criativo-expressivas apresenta 22 códigos agrupados em 5 categorias: comunidade, suporte, cuidado e co-cuidado, melhoria de espaços e segurança. Os resultados apontam a geração de processos de resiliência ao adaptar e gerar os recursos para enfrentar a incerteza dentro do mesmo contexto escolar. Torna-se relevante a continuidade de espaços de apoio emocional para os docentes, considerando que ainda são desconhecidas as consequências psicológicas que este esforço e dedicação tem na comunidade escolar. Palavras-chave: resiliência; covid-19; confinamento; educação; saúde mental.

Containment and resilience spaces in educational communities in the context of COVID-19 in Chile

Abstract. This study aimed at identifing resilience factors in a school community, during the COVID-19 pandemic. Schools communities have had to adapt their communications and learning methods, increasing the responsibilities and workload of educators. Many teachers have reduced their perception of well-being, developed depression, frustrations feelings and low self-efficacy. Some schools have implemented interventions to support digital and emotional skills, such as the "Me cuido para cuidarnos" psychosocial program. During 2020, 35 private and subsidize schools in Chile, participating in this initiative, reaching 1.599 teachers for 259 hours of online training. Using qualitative techniques (creative workshop and brainstorming). Results showed 5 main clusters of information: community, support, well-being, improve of space and security, concluding that the scholar community has started to generate resilience processes, using their own resources to cope with uncertainty. It's important to continue giving teachers emotional support.

Keywords: resilience; covid-19; lockdown; education; mental health. 


\section{Introducción}

Diversos medios de comunicación y resultados de investigaciones realizadas en contextos variados han señalado que la pandemia de la COVID-19 detonó una crisis mundial en la salud pública (Dey et al., 2021; Espada et al., 2020; Kaplan et al., 2020; Masten y Motti-Stefanidi, 2020). Como medida para disminuir los contagios se determinó -como política internacional (PAHO, 2020)- llevar a cabo un distanciamiento físico a través del confinamiento en los hogares, lo que transformó todos los hábitos y costumbres que regularmente se llevaban a cabo al interior de las familias (Brazendale et al., 2017). Por otra parte, la vida escolar en su forma presencial fue interrumpida al comenzar el año escolar 2020 ante el avance de la pandemia; directivos, docentes, estudiantes y sus familias se vieron obligados a poner en práctica otras formas de comunicarse y aprender en las diversas latitudes (Inga-Paida et al., 2020; Sánchez, 2020). Cada familia, en su diversidad, debió decidir el lugar en el hogar donde se asentaría la comunicación entre profesoras/es y estudiantes, poner a disposición o conseguir un computador para recibir el material de enseñanza y recoger la evidencia de aprendizaje de las/los alumnos. La conexión a internet, buena o mejor en algunos hogares, era débil o inexistente en otros. En los contextos escolares, las/los docentes, junto a sus directivos, debieron poner a prueba su capacidad de experimentar nuevas formas de relación educativa y de enseñanza con sus estudiantes, "debieron emprender el diseño de clases con formatos distintos y apostar a que lo que querían comunicar llegaría bien a sus alumnos y alumnas y que les serviría para aprender" (CIAE, 2020, p. 2).

Todo lo anterior, sumió a las personas en lo que hoy la Organización Mundial de la Salud denomina fatiga pandémica (Venegas y Leyva, 2020), la cual por supuesto se vio intensificada en aquellos profesionales que trabajan en labores específicas de servicio a otras personas. Esto generó -y continúa desarrollando-cuadros depresivos en los docentes y equipos directivos de los establecimientos educacionales: frustración, pérdida de sentido de su labor y baja en la percepción de autoeficacia (CIAE, 2020; Lloyd, 2020), lo que gatilló que se crearán en muchos establecimientos a nivel mundial, instancias de apoyo emocional y tecnológico para ellos (Sánchez, 2020).

El sistema educativo, entendido como tal, está inmerso en una cultura y en contextos sociales, políticos, religiosos (Pérez, 2004). Desde esta perspectiva, el sujeto y su espacio educativo también están determinados por variables de esta índole, que marcan su identidad (Sen, 2007). En consecuencia, las crisis y los factores resilientes no tienen un valor absoluto en sí mismos, presentando un valor relativo en cada sistema, ya sea este familiar, laboral, educativo, etcétera, por lo que vale la pena precisar lineamientos generales, así como la influencia de realidades externas y percepciones que corresponden al funcionamiento de los espacios educativos que viven la crisis de la COVID-19.

En los últimos tiempos, antes de la pandemia por la COVID-19, muchos países ya se encontraban en movimientos o revueltas sociales que daban cuenta de la inequidad en sus diferentes niveles, tales como: psicológicos, económicos, educativos y políticos, entre otros (Nava y Grigera, 2020). Todo esto generó crisis sistémicas 
previas a la pandemia, lo que hizo que algunos autores retomaran el concepto de Sindemia (Lolas, 2020), ya que observaron la presencia de múltiples crisis en una misma temporalidad en diferentes niveles.

Frentea un escenario complejo como el descrito, es necesario preguntarse cuáles pueden ser los aportes que entrega el enfoque de la resiliencia en ámbito educativo. Definiendo resiliencia como proceso de construcción social en el que median variables personales y ambientales (Madariaga, 2014), y dinamismo que produce cambios en los sistemas (Forés y Grané, 2008; Hurtubia, 2020; Manciaux, 2003; Rutter, 2012; Sánchez y Gutiérrez, 2016; Ungar, 2012; Vanistendael y Lecomte, 2006).

La escuela es un sistema complejo (Day y Gu, 2015; Pérez, 2004) y como tal, puede ser sujeto de resiliencia, como sostiene Masten "la resiliencia es la capacidad de un sistema de adaptarse, con éxito, frente a las amenazas y los riesgos que ponen en peligro su función, desarrollo o viabilidad [...] El concepto se puede aplicar a varios tipos de sistemas, con distintos niveles de interacción, entre ellos; un microorganismo, un niño, una familia, un sistema de seguridad, un sistema económico, o el cambio climático" (2014, p. 6). A raíz de una sindemia o una crisis de gran magnitud, es evidente que se transforme la cotidianeidad y, por tanto, la vida escolar. La actual pandemia no sólo ha exigido nuevas formas de reestructurar el sistema educativo, sin preparación previa, sino que representa además de una crisis mundial de salud pública y económica, también una de bienestar familiar y de funcionamiento psicológico, sobre todo en los largos períodos de confinamiento y la incertidumbre que persiste hasta el día de hoy (Barzilay et al., 2020). La complejidad ha sido entonces al menos por tres vías: la primera, al no contar con los conocimientos e insumos básicos para la tele-educación; la segunda, que como nunca antes se debió trabajar codo a codo con madres, padres y apoderados para lograr el proceso de enseñanza/aprendizaje (o sea, una parentalidad activa en la educación de los niños, niñas y jóvenes); y, la tercera, la cronicidad de esta crisis sanitaria, extendida por más de un año.

Frente al contexto previamente descrito, la Fundación para el Servicio del Perfeccionamiento de la Educación Católica (SEPEC) comprendió la necesidad de ofrecer herramientas innovadoras que ayudasen a la escuela a innovar sus modelos y estrategias de acción, desarrollando un programa formativo llamado "Me cuido para cuidarnos", en el cual, a partir del enfoque de la resiliencia, se co-costruyeron herramientas y estrategias adecuadas para generar respuestas innovadoras en tiempos de crisis, promoviendo un espacio de contención de los equipos educativos.

Este programa formativo involucró 35 escuelas chilenas privadas y subvencionadas, 1.599 participantes por un total de 259 horas de formación online. Cabe señalar que los establecimientos están ubicados en sectores geográficos muy diferentes y en realidades socioeconómicas y culturales también distintas, desde algunos en sectores de alta vulnerabilidad social a otros en espacios más acomodados, lo que hace que los resultados evidencien un mapa bastante interesante del sistema escolar básico y medio en Chile el año 2020.

A partir del material generado durante la formación se organizó el presente estudio, analizando desde el paradigma de la resiliencia, los elementos que contribuyen a la generación u obstaculización de escuelas resilientes, frente al escenario complejo desencadenado por la crisis socio-sanitaria de la COVID-19. Los resultados 
representan, desde una mirada cualitativa, las opiniones de los docentes en relación a los aprendizajes y desafíos que propone la promoción de la resiliencia en los entornos educativos hoy en día.

\section{Metodología}

\subsection{Muestra}

En total, participaron 1.599 sujetos pertenecientes a 35 escuelas chilenas, privadas y subvencionadas, miembros de la red de colegios de la Vicaría de la Educación del Arzobispado de Santiago, a través de un programa formativo voluntario de 8 horas online (total 259 horas), promovido por la SEPEC con el objetivo de apoyar al personal educativo, frente al aumento de la carga laboral y estrés a consecuencia del cambio en la modalidad de enseñanza de clases presenciales a online. Durante la formación se trataron temas como: el afrontamiento de la adversidad desde una perspectiva resiliente; estrategias de autocuidado y fortalecimiento de vínculos en contextos de confinamiento; y, la generación de escuelas resilientes frente a nuevos desafíos.

\subsection{Instrumento}

Durante la formación online se realizaron actividades creativo-expresivas con el objetivo de suscitar los procesos de reflexión entre los asistentes, a través de tres preguntas inspiradoras, que gatillaron la interacción y colaboración entre quienes participaron de la actividad llevada a cabo de manera sincrónica. Cada pregunta se correlacionó con una actividad creativo-expresiva, tales preguntas fueron: ¿cómo tener una escuela más resiliente que cuide a sus estudiantes y a toda la comunidad?, ¿con qué elementos ya se cuenta? y¿cuáles son necesarios promover o introducir?

Cada actividad creativo-expresiva fue desarrollada en grupos de trabajo de cinco personas; durante 45 minutos cada grupo realizó un brainstorming a partir de la pregunta generadora, para luego crear un producto final (collage, infografía, etc.), utilizando recursos de la web (pantallas colaborativas, Canvas, etc.). Finalizado el tiempo cada grupo expuso su producto y las principales ideas en plenario.

\subsection{Procedimiento y análisis}

La recolección de los datos cualitativos se realizó por medio de una serie de actividades creativo-expresivas. Los materiales producto de las actividades creativoexpresivas fueron expuestos en un plenario, del que tomaron parte todos los grupos de trabajo, generando un espacio en donde los participantes fueron exponiendo sus ideas y reflexionando sobre las acciones que se podían implementar a nivel individual y escolar. La discusión generada durante el plenario fue guiada por los relatores de la formación.

El presente estudio analiza una de estas actividades, relacionada con el cómo generar una escuela más resiliente que integre a los profesores, estudiantes, padres y todo el personal. Previamente se informó a las escuelas y participantes sobre el estudio y la confidencialidad de los datos. 


\section{Resultados}

Las sesiones produjeron reflexiones e ideas que fueron transcritas y sistematizadas en 22 códigos que fueron agrupados en 5 grandes categorías comprensivas. La figura 1 gráfica la relación entre códigos y categorías.

Figura 1. Red de códigos y categorías

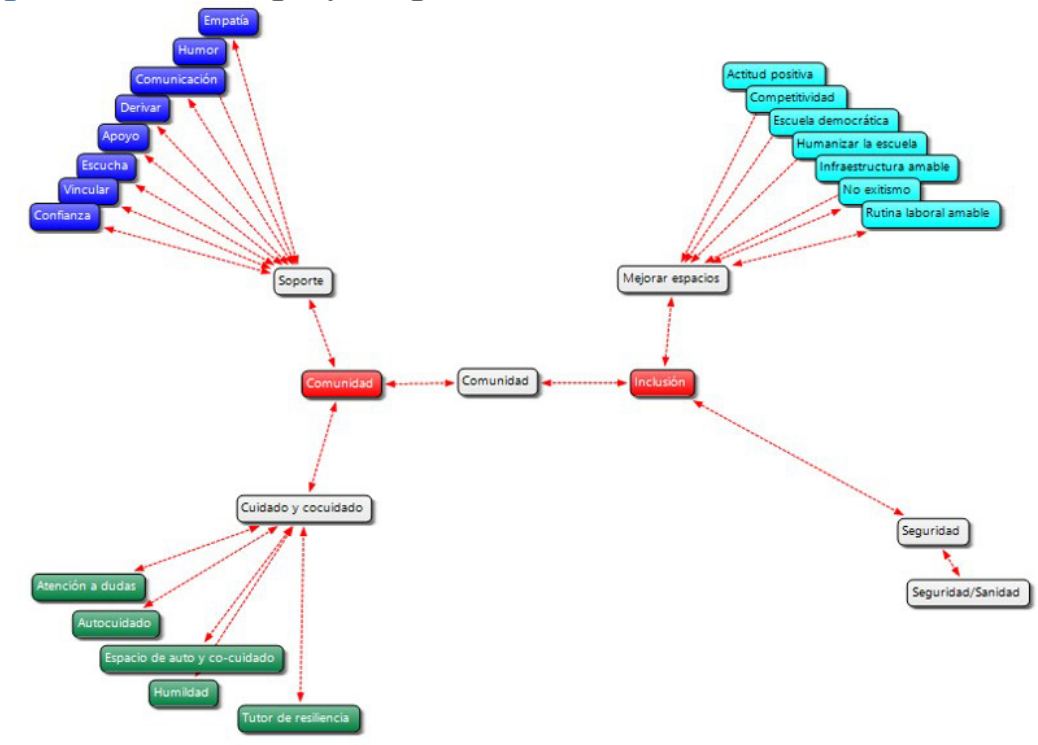

Fuente: elaboración propia

La categoría con más referencias directas se corresponde a Comunidad, en tanto que la mayor parte de las afirmaciones se relaciona con ella. Se elabora a partir de referencias directas a la comunidad educativa, su reconocimiento o su fortaleza, manifestando en ellas la importancia de esta como parte del proceso de resiliencia, así como el constante sentido de pertenencia a la misma, "Sentido de pertenencia, recuperando actividades que se perdieron como el 'bautizo' a los nuevos, que era un rito de bienvenida comunitario; o, la ceremonia de navidad" (063). "Seguir potenciando la capacidad de acogida que tiene el colegio, y apoyar como comunidad a quien lo necesita" (228).

- Cuidado y co-cuidado agrupa las referencias a actividades de cuidado de sí mismo, de la otra persona (colega, estudiante, asistente de la educación, aporderado/a, entre otros) y de la comunidad como un todo. Se respeta la referencia específica al co-cuidado. "Ver este momento como una oportunidad para pensar en cómo seguir trabajando el co-cuidado" (210). "Reforzar medidas de autocuidado y co-cuidado" (142).

- Mejorar espacios da cuenta de los de las referencias a espacios tanto físicos como simbólicos, refiriendo a espacios de tiempo y espacios psicológicos indistintamente. Se propone una mejora de los espacios haciendo de estos algo más amable para el desarrollo personal y laboral. "Humanizar la escuela (no números, si personas). No normalizar los números" (017). 
"Escuela del cuidado y del buen trato que respeta a los miembros de toda la comunidad" (255).

- Soporte: la categoría soporte da cuenta de las referencias a las tareas y actitudes de cuidados y soporte entre los miembros de la comunidad. Tales aspectos refieren tanto a intenciones de actitudes como a medidas concretas. "Actuar con empatía y asertividad en los distintos niveles (niños, profes)" (215).

- Seguridad aúna las referencias al cuidado sanitario de estudiantes y cuerpo docente, considerando protocolos y medidas sanitarias. Hace referencia a la inexorable necesidad de preparación sanitaria ante el riesgo que supone la interacción física durante la pandemia. Son medidas claras y prácticas. "Comenzar a preparase para el retorno y asegurarse de cumplir con los protocolos de seguridad; por ejemplo, reorganizar los horarios de colación"(147).

\section{Discusión}

La sistematización de los aportes entregados durante la investigación da cuenta de un proceso reactivo, resaltando una crisis que afecta a diversos niveles. Los comentarios en general apuntan a formas de enfrentarla; bien sea, basándose en idearios, como en experiencias que han dejado la percepción de un buen resultado; bien sea, apuntando a señalar los recursos considerados tanto en la observación interna del grupo como en la interacción con ambiente circundante, identificando las oportunidades como también sus carencias.

Los docentes reconocen que la crisis socio-sanitaria desencadenada por la pandemia COVID-19, ha generado una serie de cambios en sus rutinas cotidianas que impactan sobre sus vidas privadas y profesionales, viéndose obligados a adaptar sus métodos y estrategias educativas, generando dudas, miedo y estrés (Sánchez, 2020), pero también se ha reforzado el sentido de pertenencia a la escuela.

Los resultados de este estudio evidencian el reconocimiento, por parte de los docentes, de la comunidad escolar como un pilar de apoyo fundamental que debe continuar siendo reforzado, para enfrentar los desafíos generados por la COVID-19 y la modalidad de las clases online. Esto demuestra que el sistema educativo y la escuela viven en una relación de interdependencia con sus contextos (Forés y Grané, 2012; Pérez, 2004), relación que marca la identidad y el sentido de pertenencia del docente en su escuela.

En línea con el enfoque de la resiliencia, focalizado en los recursos con los que se cuenta, más que en las debilidades o carencias (Ungar, 2019; Vanistendael, 2014), puede decirse que en las respuestas de los docentes primaron los factores protectores de la resiliencia por los factores de riesgo, reconociendo la complejidad y dificultad de la situación, pero resignificándola como un aprendizaje o crecimiento para la persona y la escuela. El proceso de resiliencia se caracteriza por la interacción constante de mecanismos o factores de riesgo y de protección, que facilitan o dificultan dicho proceso, ya que gracias a la existencia de ambos factores es posibile resiliar (Kotliarenco et al., 1999; Melillo y Suárez-Ojeda, 2001; Rutter, 1993). 
Analizando los resultados a partir de los factores de protección y riesgo para la resiliencia, encontramos que cuatro de las cinco categorías identificadas en el presente estudio, pueden estar asociadas a factores de resiliencia: comunidad, soporte, mejora de espacios, cuidado y cocuidado. Mientras que la categoría seguridad puede verse como un elemento ambivalente entre el riesgo y la protección, al englobar significados directamente relacionados con el aspecto sanitario y los protocolos de distanciamiento a favor de la prevención de la COVID-19 en la escuela.

Desde elámbito educativo, promover procesos de resiliencia requiere considerar todos los elementos que forman parte del mundo escolar (infraestructura, docentes, estudiantes, padres, etc.). El modelo de Henderson y Milstein (2003) es el que mejor se adapta a este entorno de la escuela (Day y Gu, 2015; Mateu et al., 2018; Serrano y Sanz, 2019), modelo de seis pasos dividido en acciones de mitigación del riesgo (enriquecimiento de los vínculos, establecimiento de límites, life skills) y de promoción de la resiliencia (brindar afecto, transmisión de expectativas, oportunidades de participación).

Al comparar los resultados de este estudio con el modelo de Henderson y Milsten (2003), se establece una clara relación entre las categorías arrojadas por el estudio y los pasos del modelo. Comenzado por la mitigación de los riesgos se encuentra:

- Enriquecimiento de los vínculos/comunidad: fortalecimiento de los vínculos entre todos los componentes de la escuela (estudiantes, docentes, padres, personal administrativo, etc). Acción asociada a la categoría "comunidad" que incluye aspectos de la comunidad y la inclusión de todos sus actores. Las respuestas agrupadas en esta categoría demuestran cómo los vínculos generados en el entorno escolar, se convierten en un espacio de refugio y pertenencia, frente a la realidad compleja que están viviendo los docentes. Esto es coherente con el estudio efectuado en Chile (CIAE, 2020) que ha dado cuenta; por una parte, que los docentes no se han quedado en una actitud pasiva de queja; y, por otra, en una disposición de poner el acento en aspectos socioemocionales. Además, es coherente con éste en el sentido de que para hacer comunidad debe primar un mayor sentido de colaboración entre colegas versus la competencia, debiendo a la par establecer una estrecha relación con los padres y apoderado/as, en el sentido del trabajo con todos los actores que componen la comunidad educativa. Esto último es interesante si se piensa en la heterogeneidad de los establecimientos que fueron parte de esta intervención, en el sentido de sus realidades geográficas y socioculturales. El fuerte sentido de pertenencia que tienen las escuelas involucradas en este estudio, funciona como promotor de resiliencia en los docentes y el mensaje que transmiten a los estudiantes y madres, padres y apoderados. Lo anterior, podría estar conectado con la ritualidad y la espiritualidad que promueve este grupo de escuelas, expresadas a través de hábitos que se mantuvieron en la pandemia, así como la esperanza de que la situación va a mejorar en algún momento. Cabe señalar que la espiritualidad es parte de la resiliencia (Cyrulnik, 2018; Irurzun et al., 2017; Redondo et al., 2017; Vanistendael, 2005) lo cual puede ser un elemento clave para comprensión de este resultado. 
- Establecimiento de límites/autocuidado y co-cuidado: la complejidad del mundo escolar, debido a la constante interacción de relaciones, requiere el establecimiento de normas y límites claros que sean coherentes con el proyecto educativo y compartidos por toda la comunidad. Disminuyendo el riesgo de burnout por parte de los profesionales y estableciendo relaciones sanas, acciones en línea con la categoría "autocuidado y co-cuidado" que engloba aspectos individuales (autocuidado), así como acciones de cuidado desde el establecimiento "co-cuidado". El establecimiento de límites aparece como una acción a favor de la salud mental del docente, acción que trae beneficios a nivel personal y comunitario. Esta categoría puede ser considerada como un "nuevo descubrimiento" por parte de los docentes a raíz de la pandemia, un aspecto ante el cual antes no ponían particular atención, pero que hoy comienza a ser valorado, tanto por los docentes como por la dirección de los establecimientos. Los docentes durante el 2020 giraron sus mayores preocupaciones en relación al mundo de sus estudiantes y sus familias, más que sí mismos Chile (CIAE, 2020), actitud coherente con el rol del docente en tanto es un profesional de ayuda. Pero, en el contexto actual de cronicidad de la pandemia, emerge la preocupación por desarrollar acciones de autocuidado, volviéndose casi un imperativo si se quiere sostener su salud mental durante el año 2021.

- Brindar afecto y apoyo/soporte: sin presencia de un vínculo afectivo es casi imposible superar la adversidad (Cyrulnik, 2001), para ello se necesita de figuras que ofrezcan afecto y apoyo de manera incondicional, tanto a docentes como a estudiantes. Elementos que se concentran en la categoría "soporte", directamente relacionada con la comunidad y el sentido de pertenencia. El soporte, apoyo o ayuda a que cada sujeto sea un aporte a la comunidad; se relaciona elementos básicos para establecer un vínculo afectivo significativo y sano: empatía, escucha activa, humor, confianza, comunicación, etc. Los participantes de la formación reconocen en las instituciones un apoyo, el cual desean que permanezca y sea reforzado más allá de la pandemia y que sea extensivo a toda la comunidad escolar. Esto último es de absoluta coherencia con lo explicitado anteriormente.

- Generación de oportunidades de participación significativas/mejora de espacios: acción focalizada en la resolución de problemas de forma horizontal, en la que participa toda la comunidad y no sólo la dirección. Se relaciona con la categoría "mejora de espacios" al considerar este espacio como un elemento físico, mental y temporal.

Otro punto de vista en relación a la resiliencia y su promoción en entorno educativos lo ofrecen (Grané y Forés, 2020), identificando acciones orientadas al desempeño de funciones ejecutivas y a la definición de un nos-otros. Este último elemento tiene una relación directa con la categoría "comunidad", inspirada en lo relacional. En la base de los procesos de resiliencia se encuentra el establecimiento de vínculos, los cuales deben ser polivinculares (Cyrulnik, 2020), compuestos por el amor, la cooperación y la compartición. Elementos que se reflejan en las cinco categorías encontradas en el estudio, pues para tejer vínculos es necesario establecer límites, cuidarse y co-cuidarse 
y ofrecer una atención activa. Como señala Mora (2013), una escuela resiliente es la que tiene en cuenta a los demás, por esto, todas las categorías del estudio parecen girar en torno a la comunidad.

En este contexto, la categoría "seguridad", se presenta como un elemento único a la pandemia. Desde un cierto punto de vista podría ser visto como un factor de resiliencia, pero también de riesgo, en el sentido que representa el temor del otro y de la misma escuela, al no tener protocolos claros, respecto a cómo comportarse dentro de la institución para prevenir el contagio.

\section{Conclusión}

La crisis sociosanitaria que gatilla la pandemia de la COVID-19 inauguró procesos de improvisación y adaptación dinámica en todos los niveles de interacción humana. Las formas de afrontamiento, tanto individual como de los sistemas complejos, se tensionaron, por lo que se debieron encontrar nuevas formas de respuesta. La escuela, como un sistema complejo compuesto de sujetos individuales y varios subsistemas familiares, laborales, educativos, etcétera, se vio altamente estresada por esta situación completamente ajena, incierta y demandante.

Los hallazgos presentados apuntan a una rápida respuesta y adaptación a la observación, búsqueda y generación de formas de afrontamiento: un proceso de resiliencia en marcha. Los sujetos, individuales o colectivos, rastrearon con rapidez en sus entornos y contextos, hallando en ellos mismos los recursos para hacer frente a la incertidumbre.

La invitación es entonces a no dicotomizar la realidad, visibilizando seguridad versus comunidad, apreciando la necesidad de tener pautas y protocolos claros de cómo relacionarse, ya sea presencial o virtualmente, no sólo en relación al no contagio de la COVID 19; sino en todos los aspectos que sean parten de la convivencia escolar, favoreciendo el establecimiento de una real comunidad, que tenga claridad en relación a sus roles y funciones, a lo que está legitimado, a lo permitido, y a todo aquello que no sea factible o que será educado; que incluya, por supuesto, no sólo a los docentes y a los directivos, sino a los estudiantes y a sus familias, generando una cultura de la colaboración que trascienda de la pandemia. Es menester, que para que esto se forje, la generación de instancias de auto y co-cuidado de manera permanente para los distintos miembros de la comunidad educativa, haciéndose cargo de todo el desgaste emocional que cada agente ha vivido durante la pandemia y además, considerando los efectos que aún se desconocen a corto y largo plazo en la salud mental de la población.

\section{Referencias}

Barzilay, R., Moore, T. M., Greenberg, D. M., DiDomenico, G. E., Brown, L. A., White, L. K., Gur, R. C. y Gur, R. E. (2020). Resilience, COVID-19-related stress, anxiety and depression during the pandemic in a large population enriched for healthcare providers. Translational Psychiatry, 10(1), 291. https://doi.org/10.1038/s41398-020-00982-4 
Brazendale, K., Beets, M. W., Weaver, R. G., Pate, R. R., Turner-McGrievy, G. M., Kaczynski, A. T., Chandler, J. L., Bohnert, A. y von Hippel, P. T. (2017). Understanding differences between summer vs. school obesogenic behaviors of children: the structured days hypothesis. International Journal of Behavioral Nutrition and Physical Activity, 14(1), 100. https://doi. org/10.1186/s12966-017-0555-2

CIAE (2020). COVID-19 Nuevos Contextos, nuevas demandas y experiencia docente en Chile. Recuperado de https://bit.ly/3eWojw7

Cyrulnik, B. (2001). La maravilla del dolor : el sentido de la resiliencia. Granica. Recuperado de https:// bit.ly/3x5WmID

Cyrulnik, B. (2018). Psicoterapia de Dios. Gedisa.

Cyrulnik, B. (2020). Escribí soles de noche. Gedisa.

Day, C.y Gu, Q. (2015). Educadores resilientes, escuelas resilientes: Construir y sostener la calidad educativa en tiempos difíciles. Narcea Ediciones.

Dey, N., Mishra, R., Fong, S. J., Santosh, K. C., Tan, S.y Crespo, R. G. (2021). COVID-19: Psychological and Psychosocial Impact, Fear, and Passion. Digital Government: Research and Practice, 2(1), 1-4. https://doi.org/10.1145/3428088

Espada, J. P., Orgilés, M., Piqueras, J. A.y Morales, A. (2020). Las Buenas Prácticas en la Atención Psicológica Infanto-juvenil ante el COVID-19. Clínica y Salud, 31(2), 109-113. https://doi. org/10.5093/clysa2020a14

Forés, A. y Grané, J. (2008). La resiliencia. Crecer desde la adversidad. Plataforma Editorial.

Forés, A. y Grané, J. (2012). La resiliencia en entorno socioeducativos. Narcea Ediciones.

Grané, J. y Forés, A. (2020). Hagamos que sus vidas sean extraordinarias. Octaedro Editorial. https:// doi.org/9788418348297

Henderson, N. y Milstein, M. (2003). Resiliencia en las escuelas. Paidós.

Hurtubia, V. (2020). Deconstruir para construir. La formación de facilitadores de resiliencia y su aporte para la construcción de una ciudadanía del siglo XXI. In R. Martínez-Rodríguez y L. Benítez Corona (Eds.), La resiliencia en la ciudadanía del siglo XXI: una perspectiva integradora (p. 154). Newton Edición y Tecnología Educativa.

Inga-Paida, M. I., Garcia-Herrera, D. G., Castro-Salazar, A. Z. y Erazo-Álvarez, J. C. (2020). Educación y Covid-19: Percepciones docentes para enfrentar la pandemia. Revista Arbitrada Interdisciplinaria Koinonía, 5(1), 310. https://doi.org/10.35381/r.k.v5i1.785

Irurzun, J. I., Mezzadra, J. y Preuss, M. (2017). Resiliencia y espiritualidad. Aportes para su estudio desde una perspectiva psicológica. Revista CientíficaArbitrada de La Fundación MenteClara, 2(2), 205-216. https://doi.org/10.32351/rca.v2.2.34

Kaplan, J., Frias, L. y McFall-Johnsen, M. (2020). A third of the global population is on coronavirus lockdown-here's our constantly updated list of countries and restrictions. Inside. Recuperado de https://bit.ly/3eYklxH

Kotliarenco, M. A., Mardones, F., Melillo, A. y Suárez-Ojeda, E. N. (1999). Actualizaciones en Resiliencia (Fundación). a Universidad Nacional de Lanús.

Lloyd, M. (2020). Desigualdades educativas y brecha digital en tiempos de covid-19. In H. Casanova (Ed.), Educación y pandemia: una visión académica (pp. 115-121). Universidad Nacional Autónoma de México, Instituto de Investigaciones sobre la Universidad y la Educación. Recuperado de https://bit.ly/3kWC8yk

Lolas, F. (2020). Perspectivas bioéticas en un mundo en sindemia. Acta Bioethica, 26(1), 7-8. https:// doi.org/10.4067/S1726-569X2020000100007

Madariaga, J. M. (2014). Nuevas miradas sobre la resiliencia : ampliando ámbitos y prácticas. Editorial Gedisa,. Recuperado de https://bit.ly/2UFRNrn

Manciaux, M. (2003). La resiliencia: resistir y rehacerse (M. Manciaux (ed.)). Gedisa.

Masten, A. S. (2014). Global Perspectives on Resilience in Children and Youth. Child Development, 85(1), 6-20. https://doi.org/10.1111/cdev.12205 
Masten, A. S. y Motti-Stefanidi, F. (2020). Multisystem Resilience for Children and Youth in Disaster: Reflections in the Context of COVID-19. Adversity and Resilience Science, 1(2), 95-106. https://doi.org/10.1007/s42844-020-00010-w

Mateu, R., García, M., Flores, R., Gil, J. y Caballer Miedes, A. (2018). Promoción de la resiliencia en las escuelas de educación especial. In B. Cyrulnik y M. Anaut (Eds.), Resiliencia y Adaptación, la familia y la escuela como tutores de resiliencia. Gedisa.

Melillo, A. y Suárez-Ojeda, E. N. (2001). Resiliencia: descubriendo las propias fortalezas. Paidós.

Mora, F. (2013). Neuroeducación, solo se puede aprender aquello que se ama. Alianza.

Nava, A. y Grigera, J. (2020). Pandemia y protesta social: Tendencias de la conflictividad social y laboral en Argentina 2019-2020. Jacobin. https://doi.org/10.1590/SciELOPreprints.1351

PAHO (2020). El bienestar y la resiliencia en la familia en el contexto de COVID - 19. Recuperado de https://bit.ly/3x3lkby

Pérez, F. (2004). El medio social como estructura psicológica, reflexiones del modelo ecológico de Bronfenbrenner. EduPsykhé: Revista de Psicología y Psicopedagogía, 3(2), 161-177.

Redondo, T., Ibañez, C. y Barbas-Abad, S. (2017). Espiritualmente resilientes. Relación entre espiritualidad y resiliencia en cuidados paliativos. Clínica y Salud, 28(3), 117-121. https:// doi.org/10.1016/j.clysa.2017.09.001

Rutter, M. (1993). Resilience: Some conceptual considerations. Journal of Adolescent Health, 14(8), 626-631. https://doi.org/10.1016/1054-139X(93)90196-V

Rutter, M. (2012). Resilience as a dynamic concept. Development and Psychopathology, 24(2), 335344. https://doi.org/10.1017/S0954579412000028

Sánchez, A. y Gutiérrez, L. (2016). Criterios de resiliencia entrevista a Boris Cyrulnik. Gedisa.

Sánchez, J. M. (2020). Intervención en línea para el aumento de la autoeficacia en habilidades docentes por Internet ante la contingencia del COVID-19. Enseñanza y Teaching: Revista Interuniversitaria de Didáctica, 38(1), 125-145. https://doi.org/10.14201/et2020381125145

Sen, A. (2007). Identity and Violence, The Ilusion of destinity. Penguin Books.

Serrano, Á. y Sanz, R. (2019). Reflections and practical proposals to develop the capacity of resilience in front of conflicts in the school. Publicaciones de La Facultad de Educacion y Humanidades Del Campus de Melilla, 49(1), 177-190. https://doi.org/10.30827/publicaciones.v49i1.9861

Ungar, M. (2012). The Social Ecology of Resilience: A handbook of theory and practice (M. Ungar (ed.)). Springer New York. https://doi.org/10.1007/978-1-4614-0586-3

Ungar, M. (2019). Designing resilience research: Using multiple methods to investigate risk exposure, promotive and protective processes, and contextually relevant outcomes for children and youth. Child Abuse and Neglect, 96(March), 104098. https://doi.org/10.1016/j. chiabu.2019.104098

Vanistendael, S. (2014). Resiliencia: el reto del cambio de mirada. In B. Cyrulnik (Ed.), Nuevas miradas sobre la resiliencia. Gedisa.

Vanistendael, S. (2005). La resiliencia: desde una inspiración hacia cambios prácticos. 2 Congreso Internacional de Los Transtornos Del Comportamiento En Niños y Adolescentes, pagina 8, 1-13.

Vanistendael, S. y Lecomte, J. (2006). La felicidad es posible. Gedisa.

Venegas, C. y Leyva, A. (2020). La fatiga y la carga mental en los teletrabajadores: a propósito del distanciamiento social. Rev Esp Salud Pública., 94(9), Recuperado de 1-17. https://bit. $\underline{\text { Iy/3ZvFIJa }}$

Como citar este artículo en APA:

Hurtubia, V., Tartakowsky, V., Acuña M. y Landoni, M. (2021). Espacios de contención y resiliencia en comunidades educativas frente al contexto de la COVID-19 en Chile. Revista Iberoamericana de Educación, 86(2), 113-123. https://doi.org/10.35362/rie8624383 